\title{
A rank formula for the self-commutators of rational Toeplitz tuples
}

\author{
In Sung Hwang ${ }^{1}$, An Hyun Kim ${ }^{2 *}$ and Sumin Kim ${ }^{1}$
}

"Correspondence:

ahkim@changwon.ac.kr

${ }^{2}$ Department of Mathematics,

Changwon National University,

Changwon, 641-773, Korea

Full list of author information is

available at the end of the article

\begin{abstract}
In this paper we derive a rank formula for the self-commutators of tuples of Toeplitz operators with matrix-valued rational symbols.
\end{abstract}

MSC: Primary 47B20; 47B35; 47A13; secondary 30H10; 47A57

Keywords: block Toeplitz operators; jointly hyponormal; bounded type functions; rational functions; self-commutators

\section{Introduction}

Let $\mathcal{H}$ and $\mathcal{K}$ be complex Hilbert spaces, let $\mathcal{B}(\mathcal{H}, \mathcal{K})$ be the set of bounded linear operators from $\mathcal{H}$ to $\mathcal{K}$, and write $\mathcal{B}(\mathcal{H}):=\mathcal{B}(\mathcal{H}, \mathcal{H})$. For $A, B \in \mathcal{B}(\mathcal{H})$, we let $[A, B]:=A B-B A$. An operator $T \in \mathcal{B}(\mathcal{H})$ is said to be normal if $\left[T^{*}, T\right]=0$, hyponormal if $\left[T^{*}, T\right] \geq 0$. For an operator $T \in \mathcal{B}(\mathcal{H})$, we write $\operatorname{ker} T$ and $\operatorname{ran} T$ for the kernel and the range of $T$, respectively. For a subset $\mathcal{M}$ of a Hilbert space $\mathcal{H}, \operatorname{cl} \mathcal{M}$ and $\mathcal{M}^{\perp}$ denote the closure and the orthogonal complement of $\mathcal{M}$, respectively. Also, let $\mathbb{T} \equiv \partial \mathbb{D}$ be the unit circle (where $\mathbb{D}$ denotes the open unit disk in the complex plane $\mathbb{C}$ ). Recall that $L^{\infty} \equiv L^{\infty}(\mathbb{T})$ is the set of bounded measurable functions on $\mathbb{T}$, that the Hilbert space $L^{2} \equiv L^{2}(\mathbb{T})$ has a canonical orthonormal basis given by the trigonometric functions $e_{n}(z)=z^{n}$, for all $n \in \mathbb{Z}$, and that the Hardy space $H^{2} \equiv H^{2}(\mathbb{T})$ is the closed linear span of $\left\{e_{n}: n \geq 0\right\}$. An element $f \in L^{2}$ is said to be analytic if $f \in H^{2}$. Let $H^{\infty}:=L^{\infty} \cap H^{2}$, i.e., $H^{\infty}$ is the set of bounded analytic functions on $\mathbb{D}$.

We review the notion of functions of bounded type and a few essential facts about Hankel and Toeplitz operators and for that we will use [1-4].

For $\varphi \in L^{\infty}$, we write

$$
\varphi_{+} \equiv P \varphi \in H^{2} \quad \text { and } \quad \varphi_{-} \equiv \overline{P^{\perp} \varphi} \in z H^{2}
$$

where $P$ and $P^{\perp}$ denote the orthogonal projection from $L^{2}$ onto $H^{2}$ and $\left(H^{2}\right)^{\perp}$, respectively. Thus we may write $\varphi=\overline{\varphi_{-}}+\varphi_{+}$. We recall that a function $\varphi \in L^{\infty}$ is said to be of bounded type (or in the Nevanlinna class $\mathcal{N}$ ) if there are functions $\psi_{1}, \psi_{2} \in H^{\infty}$ such that

$$
\varphi(z)=\frac{\psi_{1}(z)}{\psi_{2}(z)} \quad \text { for almost all } z \in \mathbb{T} .
$$

(c) 2016 Hwang et al. This article is distributed under the terms of the Creative Commons Attribution 4.0 International License (http://creativecommons.org/licenses/by/4.0/), which permits unrestricted use, distribution, and reproduction in any medium, provided you give appropriate credit to the original author(s) and the source, provide a link to the Creative Commons license, and indicate if changes were made. 
We recall [5], Lemma 3, that if $\varphi \in L^{\infty}$ then

$$
\varphi \text { is of bounded type } \Longleftrightarrow \operatorname{ker} H_{\varphi} \neq\{0\} \text {. }
$$

Assume now that both $\varphi$ and $\bar{\varphi}$ are of bounded type. Then from the Beurling's theorem, $\operatorname{ker} H_{\overline{\varphi_{-}}}=\theta_{0} H^{2}$ and $\operatorname{ker} H_{\overline{\varphi_{+}}}=\theta_{+} H^{2}$ for some inner functions $\theta_{0}, \theta_{+}$. We thus have $b:=\overline{\varphi_{-}} \theta_{0} \in H^{2}$, and hence we can write

$$
\varphi_{-}=\theta_{0} \bar{b} \text { and similarly } \varphi_{+}=\theta_{+} \bar{a} \quad \text { for some } a \in H^{2} .
$$

By Kronecker's lemma [3], p.183, if $f \in H^{\infty}$ then $\bar{f}$ is a rational function if and only if $\operatorname{rank} H_{\bar{f}}<\infty$, which implies that

$$
\bar{f} \text { is rational } \Longleftrightarrow f=\theta \bar{b} \text { with a finite Blaschke product } \theta \text {. }
$$

Let $M_{n \times r}$ denote the set of all $n \times r$ complex matrices and write $M_{n}:=M_{n \times n}$. For $\mathcal{X}$ a Hilbert space, let $L_{\mathcal{X}}^{2} \equiv L_{\mathcal{X}}^{2}(\mathbb{T})$ be the Hilbert space of $\mathcal{X}$-valued norm square-integrable measurable functions on $\mathbb{T}$ and let $L_{\mathcal{X}}^{\infty} \equiv L_{\mathcal{X}}^{\infty}(\mathbb{T})$ be the set of $\mathcal{X}$-valued bounded measurable functions on $\mathbb{T}$. We also let $H_{\mathcal{X}}^{2} \equiv H_{\mathcal{X}}^{2}(\mathbb{T})$ be the corresponding Hardy space and $H_{\mathcal{X}}^{\infty} \equiv H_{\mathcal{X}}^{\infty}(\mathbb{T})=L_{\mathcal{X}}^{\infty} \cap H_{\mathcal{X}}^{2}$. We observe that $L_{\mathbb{C}^{n}}^{2}=L^{2} \otimes \mathbb{C}^{n}$ and $H_{\mathbb{C}^{n}}^{2}=H^{2} \otimes \mathbb{C}^{n}$.

For a matrix-valued function $\Phi \equiv\left(\varphi_{i j}\right) \in L_{M_{n}}^{\infty}$, we say that $\Phi$ is of bounded type if each entry $\varphi_{i j}$ is of bounded type, and we say that $\Phi$ is rational if each entry $\varphi_{i j}$ is a rational function.

Let $\Phi \equiv\left(\varphi_{i j}\right) \in L_{M_{n}}^{\infty}$ be such that $\Phi^{*}$ is of bounded type. Then each $\bar{\varphi}_{i j}$ is of bounded type. Thus in view of (1.2), we may write $\varphi_{i j}=\theta_{i j} \bar{b}_{i j}$, where $\theta_{i j}$ is inner and $\theta_{i j}$ and $b_{i j}$ are coprime, in other words, there does not exist a nonconstant inner divisor of $\theta_{i j}$ and $b_{i j}$. Thus if $\theta$ is the least common multiple of $\left\{\theta_{i j}: i, j=1,2, \ldots, n\right\}$, then we may write

$$
\Phi=\left(\varphi_{i j}\right)=\left(\theta_{i j} \bar{b}_{i j}\right)=\left(\theta \bar{a}_{i j}\right) \equiv \theta A^{*} \quad\left(\text { where } A \equiv\left(a_{j i}\right) \in H_{M_{n}}^{2}\right) .
$$

In particular, $A(\alpha)$ is nonzero whenever $\theta(\alpha)=0$ and $|\alpha|<1$.

For $\Phi \equiv\left[\varphi_{i j}\right] \in L_{M_{n}}^{\infty}$, we write

$$
\Phi_{+}:=\left[P\left(\varphi_{i j}\right)\right] \in H_{M_{n}}^{2} \quad \text { and } \quad \Phi_{-}:=\left[P^{\perp}\left(\varphi_{i j}\right)\right]^{*} \in H_{M_{n}}^{2} .
$$

Thus we may write $\Phi=\Phi_{-}^{*}+\Phi_{+}$. However, it will often be convenient to allow the constant term in $\Phi_{-}$. Hence, if there is no confusion we may assume that $\Phi_{-}$shares the constant term with $\Phi_{+}$: in this case, $\Phi(0)=\Phi_{+}(0)+\Phi_{-}(0)^{*}$. If $\Phi=\Phi_{-}^{*}+\Phi_{+} \in L_{M_{n}}^{\infty}$ is such that $\Phi$ and $\Phi^{*}$ are of bounded type, then in view of (1.4), we may write

$$
\Phi_{+}=\theta_{1} A^{*} \quad \text { and } \quad \Phi_{-}=\theta_{2} B^{*},
$$

where $\theta_{1}$ and $\theta_{2}$ are inner functions and $A, B \in H_{M_{n}}^{2}$. In particular, if $\Phi \in L_{M_{n}}^{\infty}$ is rational then the $\theta_{i}$ can be chosen as finite Blaschke products, as we observed in (1.3). For simplicity, we write $H_{0}^{2}$ for $z H_{M_{n}}^{2}$. 
We now introduce the notion of Hankel operators and Toeplitz operators with matrixvalued symbols. If $\Phi$ is a matrix-valued function in $L_{M_{n}}^{\infty}$, then $T_{\Phi}: H_{\mathbb{C}^{n}}^{2} \rightarrow H_{\mathbb{C}^{n}}^{2}$ denotes Toeplitz operator with symbol $\Phi$ defined by

$$
T_{\Phi} f:=P_{n}(\Phi f) \quad \text { for } f \in H_{\mathbb{C}^{n}}^{2}
$$

where $P_{n}$ is the orthogonal projection of $L_{\mathbb{C}^{n}}^{2}$ onto $H_{\mathbb{C}^{n}}^{2}$. A Hankel operator with symbol $\Phi \in L_{M_{n}}^{\infty}$ is an operator $H_{\Phi}: H_{\mathbb{C}^{n}}^{2} \rightarrow H_{\mathbb{C}^{n}}^{2}$ defined by

$$
H_{\Phi} f:=J_{n} P_{n}^{\perp}(\Phi f) \quad \text { for } f \in H_{\mathbb{C}^{n}}^{2},
$$

where $P_{n}^{\perp}$ is the orthogonal projection of $L_{\mathbb{C}^{n}}^{2}$ onto $\left(H_{\mathbb{C}^{n}}^{2}\right)^{\perp}$ and $J_{n}$ denotes the unitary operator from $L_{\mathbb{C}^{n}}^{2}$ onto $L_{\mathbb{C}^{n}}^{2}$ given by $J_{n}(f)(z):=\bar{z} f(\bar{z})$ for $f \in L_{\mathbb{C}^{n}}^{2}$. For $\Phi \in L_{M_{n \times m}}^{\infty}$, write

$$
\widetilde{\Phi}(z):=\Phi^{*}(\bar{z}) .
$$

A matrix-valued function $\Theta \in H_{M_{n \times m}}^{\infty}$ is called inner if $\Theta^{*} \Theta=I_{m}$ almost everywhere on $\mathbb{T}$, where $I_{m}$ denotes the $m \times m$ identity matrix. If there is no confusion we write simply $I$ for $I_{m}$. The following basic relations can easily be derived:

$$
\begin{aligned}
& T_{\Phi}^{*}=T_{\Phi^{*}}, \quad H_{\Phi}^{*}=H_{\widetilde{\Phi}} \quad\left(\Phi \in L_{M_{n}}^{\infty}\right) ; \\
& T_{\Phi \Psi}-T_{\Phi} T_{\Psi}=H_{\Phi^{*}}^{*} H_{\Psi} \quad\left(\Phi, \Psi \in L_{M_{n}}^{\infty}\right) ; \\
& H_{\Phi} T_{\Psi}=H_{\Phi \Psi}, \quad H_{\Psi \Phi}=T_{\widetilde{\Psi}}^{*} H_{\Phi} \quad\left(\Phi \in L_{M_{n}}^{\infty}, \Psi \in H_{M_{n}}^{\infty}\right) .
\end{aligned}
$$

In 2006, Gu et al. [6] have considered the hyponormality of Toeplitz operators with matrix-valued symbols and characterized it in terms of their symbols.

Lemma 1.1 (Hyponormality of block Toeplitz operators [6]) For each $\Phi \in L_{M_{n}}^{\infty}$, let

$$
\mathcal{E}(\Phi):=\left\{K \in H_{M_{n}}^{\infty}:\|K\|_{\infty} \leq 1 \text { and } \Phi-K \Phi^{*} \in H_{M_{n}}^{\infty}\right\}
$$

Then $T_{\Phi}$ is hyponormal if and only if $\Phi$ is normal and $\mathcal{E}(\Phi)$ is nonempty.

For a matrix-valued function $\Phi \in H_{M_{n \times r}}^{2}$, we say that $\Delta \in H_{M_{n \times m}}^{2}$ is a left inner divisor of $\Phi$ if $\Delta$ is an inner matrix function such that $\Phi=\Delta A$ for some $A \in H_{M_{m \times r}}^{2}$. We also say that two matrix functions $\Phi \in H_{M_{n \times r}}^{2}$ and $\Psi \in H_{M_{n \times m}}^{2}$ are left coprime if the only common left inner divisor of both $\Phi$ and $\Psi$ is a unitary constant, and that $\Phi \in H_{M_{n \times r}}^{2}$ and $\Psi \in H_{M_{m \times r}}^{2}$ are right coprime if $\widetilde{\Phi}$ and $\widetilde{\Psi}$ are left coprime. Two matrix functions $\Phi$ and $\Psi$ in $H_{M_{n}}^{2}$ are said to be coprime if they are both left and right coprime. We note that if $\Phi \in H_{M_{n}}^{2}$ is such that $\operatorname{det} \Phi \neq 0$, then any left inner divisor $\Delta$ of $\Phi$ is square, i.e., $\Delta \in H_{M_{n}}^{2}$ (cf. [7]). If $\Phi \in H_{M_{n}}^{2}$ is such that $\operatorname{det} \Phi \neq 0$, then we say that $\Delta \in H_{M_{n}}^{2}$ is a right inner divisor of $\Phi$ if $\widetilde{\Delta}$ is a left inner divisor of $\widetilde{\Phi}$.

Let $\left\{\Theta_{i} \in H_{M_{n}}^{\infty}: i \in J\right\}$ be a family of inner matrix functions. The greatest common left inner divisor $\Theta_{d}$ and the least common left inner multiple $\Theta_{m}$ of the family $\left\{\Theta_{i} \in H_{M_{n}}^{\infty}\right.$ : 
$i \in J\}$ are the inner functions defined by

$$
\Theta_{d} H_{\mathbb{C}^{p}}^{2}=\bigvee_{i \in J} \Theta_{i} H_{\mathbb{C}^{n}}^{2} \quad \text { and } \quad \Theta_{m} H_{\mathbb{C}^{q}}^{2}=\bigcap_{i \in J} \Theta_{i} H_{\mathbb{C}^{n}}^{2}
$$

Similarly, the greatest common right inner divisor $\Theta_{d}^{\prime}$ and the least common right inner multiple $\Theta_{m}^{\prime}$ of the family $\left\{\Theta_{i} \in H_{M_{n}}^{\infty}: i \in J\right\}$ are the inner functions defined by

$$
\widetilde{\Theta}_{d}^{\prime} H_{\mathbb{C}^{r}}^{2}=\bigvee_{i \in J} \widetilde{\Theta}_{i} H_{\mathbb{C}^{n}}^{2} \quad \text { and } \quad \widetilde{\Theta}_{m}^{\prime} H_{\mathbb{C}^{s}}^{2}=\bigcap_{i \in J} \widetilde{\Theta}_{i} H_{\mathbb{C}^{n}}^{2}
$$

The Beurling-Lax-Halmos theorem guarantees that $\Theta_{d}$ and $\Theta_{m}$ exist and are unique up to a unitary constant right factor, and $\Theta_{d}^{\prime}$ and $\Theta_{m}^{\prime}$ are unique up to a unitary constant left factor. We write

$$
\begin{aligned}
& \Theta_{d}=\text { left-g.c.d. }\left\{\Theta_{i}: i \in J\right\}, \quad \Theta_{m}=\text { left-l.c.m. }\left\{\Theta_{i}: i \in J\right\}, \\
& \Theta_{d}^{\prime}=\text { right-g.c.d. }\left\{\Theta_{i}: i \in J\right\}, \quad \Theta_{m}^{\prime}=\text { right-l.c.m. }\left\{\Theta_{i}: i \in J\right\} .
\end{aligned}
$$

If $n=1$, then left-g.c.d. $\{\cdot\}=$ right-g.c.d. $\{\cdot\}$ (simply denoted g.c.d. $\{\cdot\})$ and left-l.c.m. $\{\cdot\}=$ right-l.c.m. $\{\cdot\}$ (simply denoted l.c.m. $\{\cdot\}$ ). In general, it is not true that left-g.c.d. $\{\cdot\}=$ right-g.c.d. $\{\cdot\}$ and left-l.c.m. $\{\cdot\}=$ right-l.c.m. $\{\cdot\}$.

If $\theta$ is an inner function we write $I_{\theta}$ for $\theta I_{n}$ and $\mathcal{Z}(\theta)$ for the set of all zeros of $\theta$.

Lemma 1.2 Let $\Theta_{i}:=I_{\theta_{i}}$ for an inner function $\theta_{i}(i \in J)$.

(a) left-g.c.d. $\left\{\Theta_{i}: i \in J\right\}=$ right-g.c.d. $\left\{\Theta_{i}: i \in J\right\}=I_{\theta_{d}}$, where $\theta_{d}=$ g.c.d. $\left\{\theta_{i}: i \in J\right\}$.

(b) left-l.c.m. $\left\{\Theta_{i}: i \in J\right\}=$ right-l.c.m. $\left\{\Theta_{i}: i \in J\right\}=I_{\theta_{m}}$, where $\theta_{m}=$ l.c.m. $\left\{\theta_{i}: i \in J\right\}$.

Proof See [7], Lemma 2.1.

In view of Lemma 1.2, if $\Theta_{i}=I_{\theta_{i}}$ for an inner function $\theta_{i}(i \in J)$, we can define the greatest common inner divisor $\Theta_{d}$ and the least common inner multiple $\Theta_{m}$ of the $\Theta_{i}$ by

$$
\Theta_{d} \equiv \text { g.c.d. }\left\{\Theta_{i}: i \in J\right\}:=I_{\theta_{d}} \text {, where } \theta_{d}=\text { g.c.d. }\left\{\theta_{i}: i \in J\right\}
$$

and

$$
\Theta_{m} \equiv \text { l.c.m. }\left\{\Theta_{i}: i \in J\right\}:=I_{\theta_{m}} \text {, where } \theta_{m}=\text { l.c.m. }\left\{\theta_{i}: i \in J\right\} \text {. }
$$

Both $\Theta_{d}$ and $\Theta_{m}$ are diagonal-constant inner functions, i.e., diagonal inner functions, and constant along the diagonal.

By contrast with scalar-valued functions, in (1.4), $I_{\theta}$ and $A$ need not be (right) coprime. If $\Omega=$ left-g.c.d. $\left\{I_{\theta}, A\right\}$ in the representation (1.4), that is,

$$
\Phi=\theta A^{*},
$$

then $I_{\theta}=\Omega \Omega_{\ell}$ and $A=\Omega A_{\ell}$ for some inner matrix $\Omega_{\ell}$ (where $\Omega_{\ell} \in H_{M_{n}}^{2}$ because $\operatorname{det}\left(I_{\theta}\right) \neq$ 0 ) and some $A_{l} \in H_{M_{n}}^{2}$. Therefore if $\Phi^{*} \in L_{M_{n}}^{\infty}$ is of bounded type then we can write

$$
\Phi=A_{\ell}{ }^{*} \Omega_{\ell}, \quad \text { where } A_{\ell} \text { and } \Omega_{\ell} \text { are left coprime. }
$$


In this case, $A_{\ell}^{*} \Omega_{\ell}$ is called the left coprime factorization of $\Phi$ and write, briefly,

$$
\Phi=A_{\ell}^{*} \Omega_{\ell} \quad \text { (left coprime). }
$$

Similarly, we can write

$$
\Phi=\Omega_{r} A_{r}^{*}, \quad \text { where } A_{r} \text { and } \Omega_{r} \text { are right coprime. }
$$

In this case, $\Omega_{r} A_{r}^{*}$ is called the right coprime factorization of $\Phi$ and we write, succinctly,

$$
\Phi=\Omega_{r} A_{r}^{*} \quad \text { (right coprime) }
$$

In this case, we define the degree of $\Phi$ by

$$
\operatorname{deg}(\Phi):=\operatorname{dim} \mathcal{H}\left(\Omega_{r}\right)
$$

where $\mathcal{H}(\Theta):=H_{\mathbb{C}^{n}}^{2} \ominus \Theta H_{\mathbb{C}^{n}}^{2}$ for an inner function $\Theta$. It was known (cf. [8], Lemma 3.3) that if $\theta$ is a finite Blaschke product then $I_{\theta}$ and $A \in H_{M_{n}}^{2}$ are left coprime if and only if they are right coprime. In this viewpoint, in (1.10) and (1.12), $\Omega_{\ell}$ or $\Omega_{r}$ is $I_{\theta}(\theta$ a finite Blaschke product) then we shall write

$$
\Phi=\theta A^{*} \quad \text { (coprime) }
$$

On the other hand, we recall that an operator $T \in \mathcal{B}(\mathcal{H})$ is said to be subnormal if $T$ has a normal extension, i.e., $T=\left.N\right|_{\mathcal{H}}$, where $N$ is a normal operator on some Hilbert space $\mathcal{K} \supseteq \mathcal{H}$ such that $\mathcal{H}$ is invariant for $N$. The Bram-Halmos criterion for subnormality [9, 10] states that an operator $T \in \mathcal{B}(\mathcal{H})$ is subnormal if and only if $\sum_{i, j}\left(T^{i} x_{j}, T^{j} x_{i}\right) \geq 0$ for all finite collections $x_{0}, x_{1}, \ldots, x_{k} \in \mathcal{H}$. It is easy to see that this is equivalent to the following positivity test:

$$
\left(\begin{array}{cccc}
{\left[T^{*}, T\right]} & {\left[T^{* 2}, T\right]} & \ldots & {\left[T^{* k}, T\right]} \\
{\left[T^{*}, T^{2}\right]} & {\left[T^{* 2}, T^{2}\right]} & \ldots & {\left[T^{* k}, T^{2}\right]} \\
\vdots & \vdots & \ddots & \vdots \\
{\left[T^{*}, T^{k}\right]} & {\left[T^{* 2}, T^{k}\right]} & \ldots & {\left[T^{* k}, T^{k}\right]}
\end{array}\right) \geq 0 \quad(\text { all } k \geq 1)
$$

Condition (1.13) provides a measure of the gap between hyponormality and subnormality. In fact the positivity condition (1.13) for $k=1$ is equivalent to the hyponormality of $T$, while subnormality requires the validity of (1.13) for all $k$. For $k \geq 1$, an operator $T$ is said to be k-hyponormal if $T$ satisfies the positivity condition (1.13) for a fixed $k$. Thus the Bram-Halmos criterion can be stated thus: $T$ is subnormal if and only if $T$ is $k$-hyponormal for all $k \geq 1$. The notion of $k$-hyponormality has been considered by many authors aiming at understanding the bridge between hyponormality and subnormality. In view of (1.13), between hyponormality and subnormality there exists a whole slew of increasingly stricter conditions, each expressible in terms of the joint hyponormality of the tuples $\left(I, T, T^{2}, \ldots, T^{k}\right)$. Given an $n$-tuple $\mathbf{T}=\left(T_{1}, \ldots, T_{n}\right)$ of operators on $\mathcal{H}$, we let 
$\left[\mathbf{T}^{*}, \mathbf{T}\right] \in \mathcal{B}(\mathcal{H} \oplus \cdots \oplus \mathcal{H})$ denote the self-commutator of $\mathbf{T}$, defined by

$$
\left[\mathbf{T}^{*}, \mathbf{T}\right]:=\left(\begin{array}{cccc}
{\left[T_{1}^{*}, T_{1}\right]} & {\left[T_{2}^{*}, T_{1}\right]} & \ldots & {\left[T_{n}^{*}, T_{1}\right]} \\
{\left[T_{1}^{*}, T_{2}\right]} & {\left[T_{2}^{*}, T_{2}\right]} & \ldots & {\left[T_{n}^{*}, T_{2}\right]} \\
\vdots & \vdots & \ddots & \vdots \\
{\left[T_{1}^{*}, T_{n}\right]} & {\left[T_{2}^{*}, T_{n}\right]} & \ldots & {\left[T_{n}^{*}, T_{n}\right]}
\end{array}\right)
$$

By analogy with the case $n=1$, we shall say $[11,12]$ that $\mathbf{T}$ is jointly hyponormal (or simply, hyponormal) if $\left[\mathbf{T}^{*}, \mathbf{T}\right] \geq 0$, i.e., $\left[\mathbf{T}^{*}, \mathbf{T}\right]$ is a positive-semidefinite operator on $\mathcal{H} \oplus \cdots \oplus \mathcal{H}$.

Tuples $\mathbf{T} \equiv\left(T_{\Phi_{1}}, \ldots, T_{\Phi_{m}}\right)$ of block Toeplitz operators $T_{\Phi_{i}}(i=1, \ldots, m)$ will be called a (block) Toeplitz tuples. Moreover, if each Toeplitz operator $T_{\Phi_{i}}$ has a symbol $\Phi_{i}$ which is a matrix-valued rational function, then the tuple $\mathbf{T} \equiv\left(T_{\Phi_{1}}, \ldots, T_{\Phi_{m}}\right)$ is called a rational Toeplitz tuple. In this paper we will derive a rank formula for the self-commutator of a rational Topelitz tuple.

\section{The results and discussion}

For an operator $S \in \mathcal{B}(\mathcal{H}), S^{\sharp} \in \mathcal{B}(\mathcal{H})$ is called the Moore-Penrose inverse of $S$ if

$$
S S^{\sharp} S=S, \quad S^{\sharp} S S^{\sharp}=S^{\sharp}, \quad\left(S^{\sharp} S\right)^{*}=S^{\sharp} S, \quad \text { and } \quad\left(S S^{\sharp}\right)^{*}=S S^{\sharp} \text {. }
$$

It is well known [13], Theorem 8.7.2, that if an operator $S$ on a Hilbert space has a closed range then $S$ has a Moore-Penrose inverse. Moreover, the Moore-Penrose inverse is unique whenever it exists. On the other hand, it is well known that if

$$
S:=\left[\begin{array}{ll}
A & B \\
B^{*} & C
\end{array}\right] \quad \text { on } \mathcal{H}_{1} \oplus \mathcal{H}_{2}
$$

(where the $\mathcal{H}_{j}$ are Hilbert spaces, $A \in \mathcal{B}\left(\mathcal{H}_{1}\right), C \in \mathcal{B}\left(\mathcal{H}_{2}\right)$, and $B \in \mathcal{B}\left(\mathcal{H}_{2}, \mathcal{H}_{1}\right)$ ), then

$$
S \geq 0 \quad \Longleftrightarrow \quad A \geq 0, C \geq 0 \text {, and } B=A^{\frac{1}{2}} D C^{\frac{1}{2}} \quad \text { for some contraction } D ;
$$

moreover, in [14], Lemma 1.2, and [15], Lemma 2.1, it was shown that if $A \geq 0, C \geq 0$, and $\operatorname{ran} A$ is closed then

$$
S \geq 0 \quad \Longleftrightarrow \quad B^{*} A^{\sharp} B \leq C \text { and } \operatorname{ran} B \subseteq \operatorname{ran} A,
$$

or equivalently [12], Lemma 1.4,

$$
|\langle B g, f\rangle|^{2} \leq\langle A f, f\rangle\langle C g, g\rangle \quad \text { for all } f \in \mathcal{H}_{1}, g \in \mathcal{H}_{2}
$$

and furthermore, if both $A$ and $C$ are of finite rank then

$$
\operatorname{rank} S=\operatorname{rank} A+\operatorname{rank}\left(C-B^{*} A^{\sharp} B\right) .
$$

In fact, if $A \geq 0$ and $\operatorname{ran} A$ is closed then we can write

$$
A=\left[\begin{array}{cc}
A_{0} & 0 \\
0 & 0
\end{array}\right]:\left[\begin{array}{l}
\operatorname{ran} A \\
\operatorname{ker} A
\end{array}\right] \rightarrow\left[\begin{array}{c}
\operatorname{ran} A \\
\operatorname{ker} A
\end{array}\right],
$$


so that the Moore-Penrose inverse of $A$ is given by

$$
A^{\sharp}=\left[\begin{array}{cc}
\left(A_{0}\right)^{-1} & 0 \\
0 & 0
\end{array}\right] .
$$

Proposition 2.1 If $A \in \mathcal{B}(\mathcal{H})$ has a closed range then $A\left(A^{*} A\right)^{\sharp} A^{*}$ is the orthogonal projection onto $\operatorname{ran} A$.

Proof Suppose $A \in \mathcal{B}(\mathcal{H})$ has a closed range. Then (2.5) can be written as

$$
\left(P_{\operatorname{ran} A} A P_{\operatorname{ran} A}\right)^{-1}=P_{\operatorname{ran} A} A^{\sharp} P_{\operatorname{ran} A} .
$$

Since by assumption, $A^{*} A$ has also a closed range, there exists the Moore-Penrose inverse $\left(A^{*} A\right)^{\sharp}$. Observe

$$
\left(A\left(A^{*} A\right)^{\sharp} A^{*}\right)\left(A\left(A^{*} A\right)^{\sharp} A^{*}\right)=A\left(A^{*} A\right)^{\sharp} A^{*}
$$

and

$$
\left(A\left(A^{*} A\right)^{\sharp} A^{*}\right)^{*}=A\left(A^{*} A\right)^{\sharp} A^{*},
$$

which implies that $A\left(A^{*} A\right)^{\sharp} A^{*}$ is an orthogonal projection. Put

$$
K:=\operatorname{ran} A^{*} A=\operatorname{ran} A^{*}=(\operatorname{ker} A)^{\perp} .
$$

We then have

$$
\begin{aligned}
A\left(A^{*} A\right)^{\sharp} A^{*} & =A P_{K}\left(A^{*} A\right)^{\sharp} P_{K} A^{*} \\
& =A\left(P_{K}\left(A^{*} A\right) P_{K}\right)^{-1} A^{*} \quad(\text { by }(2.5)),
\end{aligned}
$$

which implies that $\operatorname{ran}\left(A\left(A^{*} A\right)^{\sharp} A^{*}\right)=\operatorname{ran} A$.

In the sequel we often encounter the following matrix:

$$
S:=\left[\begin{array}{cc}
A^{*} A & A^{*} B \\
B^{*} A & {\left[B^{*}, B\right]}
\end{array}\right],
$$

where $A$ has a closed range. If $S \geq 0$ and if $A$ and $\left[B^{*}, B\right]$ are of finite rank then by (2.4), we have

$$
\operatorname{rank} S=\operatorname{rank}\left(A^{*} A\right)+\operatorname{rank}\left(\left[B^{*}, B\right]-B^{*} A\left(A^{*} A\right)^{\sharp} A^{*} B\right) .
$$

Thus, if we write $P_{K}$ for the orthogonal projection onto $K:=\operatorname{ran} A$, then by Proposition 2.1 we have

$$
\begin{aligned}
\operatorname{rank} S & =\operatorname{rank}\left(A^{*}\right)+\operatorname{rank}\left(\left[B^{*}, B\right]-B^{*} P_{K} B\right) \\
& =\operatorname{rank}\left(A^{*}\right)+\operatorname{rank}\left(B^{*} P_{K^{\perp}} B-B B^{*}\right) .
\end{aligned}
$$


If $\Phi, \Psi \in L_{M_{n}}^{\infty}$, then by (1.7),

$$
\left[T_{\Phi}, T_{\Psi}\right]=H_{\Psi^{*}}^{*} H_{\Phi}-H_{\Phi *}^{*} H_{\Psi}+T_{\Phi \Psi-\Psi \Phi}
$$

Since the normality of $\Phi$ is a necessary condition for the hyponormality of $T_{\Phi}$ ( $c f$. [15]), the positivity of $H_{\Phi^{*}}^{*} H_{\Phi^{*}}-H_{\Phi}^{*} H_{\Phi}$ is an essential condition for the hyponormality of $T_{\Phi}$. If $\Phi \in L_{M_{n}}^{\infty}$, the pseudo-self-commutator of $T_{\Phi}$ is defined by

$$
\left[T_{\Phi}^{*}, T_{\Phi}\right]_{p}:=H_{\Phi^{*}}^{*} H_{\Phi^{*}}-H_{\Phi}^{*} H_{\Phi}
$$

Then $T_{\Phi}$ is said to be pseudo-hyponormal if $\left[T_{\Phi}^{*}, T_{\Phi}\right]_{p} \geq 0$. We also see that if $\Phi \in L_{M_{n}}^{\infty}$ then $\left[T_{\Phi}^{*}, T_{\Phi}\right]=\left[T_{\Phi}^{*}, T_{\Phi}\right]_{p}+T_{\Phi^{*} \Phi-\Phi \Phi^{*}}$.

Proposition 2.2 Let $\Phi \equiv \Phi_{-}^{*}+\Phi_{+} \in L_{M_{n}}^{\infty}$ be such that $\Phi$ and $\Phi^{*}$ are of bounded type. Thus in view of (1.4), we may write

$$
\Phi_{+}=\theta_{1} A^{*} \text { and } \Phi_{-}=\theta_{2} B^{*},
$$

where $\theta_{1}$ and $\theta_{2}$ are inner functions and $A, B \in H_{M_{n}}^{2}$. If $T_{\Phi}$ is hyponormal then $\theta_{2}$ is an inner divisor of $\theta_{1}$, i.e., $\theta_{1}=\theta_{0} \theta_{2}$ for some inner function $\theta_{0}$.

Proof See [7], Proposition 3.2.

In view of Proposition 2.2, when we study the hyponormality of block Toeplitz operators with bounded type symbols $\Phi$ (i.e., $\Phi$ and $\Phi^{*}$ are of bounded type) we may assume that the symbol $\Phi \equiv \Phi_{-}^{*}+\Phi_{+} \in L_{M_{n}}^{\infty}$ is of the form

$$
\Phi_{+}=\theta_{0} \theta_{1} A^{*} \text { and } \Phi_{-}=\theta_{0} B^{*}
$$

where $\theta_{0}$ and $\theta_{1}$ are inner functions and $A, B \in H_{M_{n}}^{2}$.

We first observe that if $\mathbf{T}=\left(T_{\varphi}, T_{\psi}\right)$ then the self-commutator of $\mathbf{T}$ can be expressed as

$$
\left[\mathbf{T}^{*}, \mathbf{T}\right]=\left[\begin{array}{ll}
{\left[T_{\varphi}^{*}, T_{\varphi}\right]} & {\left[T_{\psi}^{*}, T_{\varphi}\right]} \\
{\left[T_{\varphi}^{*}, T_{\psi}\right]} & {\left[T_{\psi}^{*}, T_{\psi}\right]}
\end{array}\right]=\left[\begin{array}{ll}
H_{\overline{\varphi_{+}}}^{*} H_{\overline{\varphi_{+}}}-H_{\overline{\varphi_{-}}}^{*} H_{\overline{\varphi_{-}}} & H_{\overline{\varphi_{+}}}^{*} H_{\overline{\psi_{+}}}-H_{\overline{\psi_{-}}}^{*} H_{\overline{\varphi_{-}}} \\
H_{\overline{\psi_{+}}}^{*} H_{\overline{\varphi_{+}}}-H_{\overline{\varphi_{-}}}^{*} H_{\overline{\psi_{-}}} & H_{\overline{\psi_{+}}}^{*} H_{\overline{\psi_{+}}}-H_{\overline{\psi_{-}}}^{*} H_{\overline{\psi_{-}}}^{*}
\end{array}\right] .
$$

For a block Toeplitz pair $\mathbf{T} \equiv\left(T_{\Phi}, T_{\Psi}\right)$, the pseudo-commutator of $\mathbf{T}$ is defined by

$$
\begin{aligned}
{\left[\mathbf{T}^{*}, \mathbf{T}\right]_{p} } & :=\left[\begin{array}{ll}
{\left[T_{\Phi}^{*}, T_{\Phi}\right]_{p}} & {\left[T_{\Psi}^{*}, T_{\Phi}\right]_{p}} \\
{\left[T_{\Phi}^{*}, T_{\Psi}\right]_{p}} & {\left[T_{\Psi}^{*}, T_{\Psi}\right]_{p}}
\end{array}\right] \\
& =\left[\begin{array}{ll}
H_{\Phi_{+}^{*}}^{*} H_{\Phi_{+}^{*}}-H_{\Phi_{*}^{*}}^{*} H_{\Phi_{-}^{*}} & H_{\Phi_{+}^{*}}^{*} H_{\Psi_{+}^{*}}-H_{\Psi_{-}^{*}}^{*} H_{\Phi_{-}^{*}} \\
H_{\Psi_{+}^{*}}^{*} H_{\Phi_{+}^{*}}-H_{\Phi_{-}^{*}}^{*} H_{\Psi_{-}^{*}} & H_{\Psi_{+}^{*}}^{*} H_{\Psi_{+}^{*}}-H_{\Psi_{-}^{*}}^{*} H_{\Psi_{-}^{*}}
\end{array}\right] .
\end{aligned}
$$

Let $\Phi_{i} \in L_{M_{n}}^{\infty}(i=1,2, \ldots, m)$ be normal and mutually commuting and let $\sigma$ be a permutation on $\{1,2, \ldots, m\}$. Then evidently,

$$
\begin{aligned}
\mathbf{T}:= & \left(T_{\Phi_{1}}, \ldots, T_{\Phi_{m}}\right) \text { is hyponormal } \\
& \Longleftrightarrow \quad \mathbf{T}_{\sigma}:=\left(T_{\Phi_{\sigma(1)}}, \ldots, T_{\Phi_{\sigma(m)}}\right) \text { is hyponormal. }
\end{aligned}
$$


Moreover, we have

$$
\operatorname{rank}\left[\mathbf{T}^{*}, \mathbf{T}\right]=\operatorname{rank}\left[\mathbf{T}_{\sigma}^{*}, \mathbf{T}_{\sigma}\right]
$$

For every $m_{0} \leq m$, let $\mathbf{T}_{m_{0}}:=\left(T_{\Phi_{1}}, \ldots, T_{\Phi_{m_{0}}}\right)$. Since

$$
\left[\mathbf{T}^{*}, \mathbf{T}\right]=\left[\begin{array}{cc}
{\left[\mathbf{T}_{\Phi_{m_{0}}}^{*}, \mathbf{T}_{\Phi_{m_{0}}}\right]} & * \\
* & *
\end{array}\right],
$$

we can see that if $\mathbf{T}$ is hyponormal then in view of (2.10), every sub-tuple of $\mathbf{T}$ is hyponormal.

We then have the following.

Lemma 2.3 Let $\Phi_{i} \in L_{M_{n}}^{\infty}$ be normal and mutually commuting. Let $\mathbf{T} \equiv\left(T_{\Phi_{1}}, \ldots, T_{\Phi_{m}}\right)$ and $\mathbf{S} \equiv\left(T_{\Lambda_{1} \Phi_{1}}, \ldots, T_{\Lambda_{m} \Phi_{m}}\right)$, where the $\Lambda_{i}$ are mutually commuting and are invertible constant normal matrices commuting with $\Phi_{j}$ and $\Lambda_{j}$ for each $i, j=1,2, \ldots, m$. Then

$\mathbf{T}$ is hyponormal $\Longleftrightarrow \mathbf{S}$ is hyponormal.

Furthermore, $\operatorname{rank}\left[\mathbf{T}^{*}, \mathbf{T}\right]=\operatorname{rank}\left[\mathbf{S}^{*}, \mathbf{S}\right]$.

Proof In view of equation (2.10), it suffices to prove the lemma when $\Lambda_{i}=I$ for all $i=$ $2, \ldots, m$. Put $\mathcal{T}:=\left[\mathbf{T}^{*}, \mathbf{T}\right]$ and $\mathcal{S}:=\left[\mathbf{S}^{*}, \mathbf{S}\right]$. Since $\Lambda_{1}$ is a constant normal matrix commuting with $\Phi_{j}$, it follows that, for all $j>1$,

$$
\begin{aligned}
\mathcal{S}_{1 j} & =H_{\left(\Lambda_{1} \Phi_{1}\right)_{+}^{*}}^{*} H_{\left(\Phi_{j}\right)_{+}^{*}}-H_{\left(\Phi_{j}\right)_{-}^{*}}^{*} H_{\left(\Lambda_{1} \Phi_{1}\right)_{-}} \\
& =H_{\left(\Phi_{1}\right)_{+}^{*} \Lambda_{1}^{*}}^{*} H_{\left(\Phi_{j}\right)_{+}^{*}}-H_{\left(\Phi_{j}\right)_{-}^{*}}^{*} H_{\Lambda_{1}\left(\Phi_{1}\right)_{-}^{*}} \\
& =T_{\Lambda_{1}} H_{\left(\Phi_{1}\right)_{+}^{*}}^{*} H_{\left(\Phi_{j}\right)_{+}^{*}}-H_{\left(\Phi_{j}\right) *}^{*} T_{\Lambda_{1}} H_{\left(\Phi_{1}\right)_{-}} \\
& =T_{\Lambda_{1}} H_{\left(\Phi_{1}\right)_{+}^{*}}^{*} H_{\left(\Phi_{j}\right)_{+}^{*}}-H_{\left(\Phi_{j}\right)_{-}^{*} \Lambda_{1}^{*}}^{*} H_{\left(\Phi_{1}\right)_{-}} \\
& =T_{\Lambda_{1}}\left(H_{\left(\Phi_{1}\right)_{+}^{*}}^{*} H_{\left(\Phi_{j}\right)_{+}^{*}}-H_{\left(\Phi_{j}\right)_{-}^{*}}^{*} H_{\left(\Phi_{1}\right)_{-}}\right) \\
& =T_{\Lambda_{1}} \mathcal{T}_{1 j} .
\end{aligned}
$$

Observe that

$$
\begin{aligned}
& \mathcal{S}_{11}=H_{\left(\Lambda_{1} \Phi_{1}\right)_{+}^{*}}^{*} H_{\left(\Lambda_{1} \Phi_{1}\right)_{+}^{*}}-H_{\left(\Lambda_{1} \Phi_{1}\right)_{-}^{*}}^{*} H_{\left(\Lambda_{1} \Phi_{1}\right)_{-}^{*}} \\
& =H_{\left(\Phi_{1}\right)_{*}^{*} \Lambda_{1}^{*}}^{*} H_{\left(\Phi_{1}\right)_{+}^{*} \Lambda_{1}^{*}}-H_{\left(\Phi_{1}\right)^{*} \Lambda_{1}^{*}}^{*} H_{\left(\Phi_{1}\right)_{-}^{*} \Lambda_{1}^{*}} \\
& =T_{\Lambda_{1}} H_{\left(\Phi_{1}\right)_{+}^{*}}^{*} H_{\left(\Phi_{1}\right)_{+}^{*}} T_{\Lambda_{1}}^{*}-T_{\Lambda_{1}} H_{\left(\Phi_{1}\right)_{-}^{*}}^{*} H_{\left(\Phi_{1}\right)_{-}^{*}} T_{\Lambda_{1}}^{*} \\
& =T_{\Lambda_{1}}\left(H_{\left(\Phi_{1}\right)_{+}^{*}}^{*} H_{\left(\Phi_{1}\right)_{+}^{*}}-H_{\left(\Phi_{1}\right)_{-}^{*}}^{*} H_{\left(\Phi_{1}\right)_{-}}\right) T_{\Lambda_{1}}^{*} \\
& =T_{\Lambda_{1}} \mathcal{T}_{11} T_{\Lambda_{1}}^{*} \text {. }
\end{aligned}
$$

Let $Q$ be the block diagonal operator with the diagonal entries $\left(T_{\Lambda_{1}}, I, \ldots, I\right)$. Then $Q$ is invertible and $\mathcal{S}=Q \mathcal{T} Q^{*}$, which gives the result. 
Lemma 2.4 Let $\mathbf{T} \equiv\left(T_{\Phi_{1}}, T_{\Phi_{2}}, \ldots T_{\Phi_{m}}\right)$, where the $\Phi_{i} \in L_{M_{n}}^{\infty}(i=1, \ldots, m)$ are normal and mutually commuting. If $\mathbf{S}:=\left(T_{\Phi_{1}-\Phi_{j_{0}}}, T_{\Phi_{2}}, \ldots T_{\Phi_{m}}\right)$ for some $j_{0}\left(2 \leq j_{0} \leq m\right)$, then

$\mathbf{T}$ is hyponormal $\Longleftrightarrow \mathbf{S}$ is hyponormal.

Furthermore, $\operatorname{rank}\left[\mathbf{T}^{*}, \mathbf{T}\right]=\operatorname{rank}\left[\mathbf{S}^{*}, \mathbf{S}\right]$.

Proof Obvious.

Corollary 2.5 Let $\Phi_{i} \in L_{M_{n}}^{\infty}(i=1, \ldots, m)$ be normal and mutually commuting. Let $\mathbf{T} \equiv$ $\left(T_{\Phi_{1}}, \ldots T_{\Phi_{m}}\right)$ and put

$$
\mathbf{S}:=\left(T_{\Phi_{1}-\Lambda_{1} \Phi_{m}}, T_{\Phi_{2}-\Lambda_{2} \Phi_{m}}, \ldots, T_{\Phi_{m-1}-\Lambda_{m-1} \Phi_{m}}, T_{\Phi_{m}}\right)
$$

where the $\Lambda_{i}(i=1, \ldots, m-1)$ are mutually commuting and are invertible constant normal matrices commuting with $\Phi_{j}$ for each $j=1, \ldots, m$. Then

$\mathbf{T}$ is hyponormal $\Longleftrightarrow \mathbf{S}$ is hyponormal.

Furthermore, $\operatorname{rank}\left[\mathbf{T}^{*}, \mathbf{T}\right]=\operatorname{rank}\left[\mathbf{S}^{*}, \mathbf{S}\right]$.

Proof This follows from Lemmas 2.3 and 2.4.

We now have the following.

Theorem 2.6 Let $\Phi_{i} \in H_{M_{n}}^{\infty}(i=1,2, \ldots, m-1)$ be mutually commuting and normal rational functions of the form

$$
\left.\Phi_{i}=A_{i}^{*} \Theta_{i} \quad \text { (left coprime }\right)
$$

where the $\Theta_{i}$ are inner matrix functions and $\Phi_{m} \equiv\left(\Phi_{m}\right)_{-}^{*}+\left(\Phi_{m}\right)_{+} \in L_{M_{n}}^{\infty}$. If $\mathbf{T}:=$ $\left(T_{\Phi_{1}}, \ldots, T_{\Phi_{m}}\right)$ is hyponormal then

$$
\operatorname{rank}\left[\mathbf{T}^{*}, \mathbf{T}\right]=\operatorname{deg}(\Theta)+\operatorname{rank}\left[T_{\Phi_{m}^{1, \Theta}}^{*}, T_{\Phi_{m}^{1, \Theta}}\right]_{p},
$$

where $\Theta:=$ right-l.c.m. $\left\{\Theta_{i}: i=1,2, \ldots, m-1\right\}$ and $\Phi_{m}^{1, \Theta}:=\left(\Phi_{m}\right)_{-}^{*}+P_{H_{0}^{2}}\left(\left(\Phi_{m}\right)_{+} \Theta^{*}\right)$.

Proof Let $\mathbf{H}_{\Phi^{*}}:=\left(H_{\Phi_{1}^{*}}, \ldots, H_{\Phi_{m-1}^{*}}\right)$. Since $\Phi_{i} \equiv\left(\Phi_{i}\right)_{+} \in H_{M_{n}}^{\infty}(i=1,2, \ldots, m-1), \mathbf{T}$ is hyponormal if and only if

$$
\left[\mathbf{T}^{*}, \mathbf{T}\right]=\left[\begin{array}{cc}
\mathbf{H}_{\Phi^{*}}^{*} \mathbf{H}_{\Phi^{*}} & \mathbf{H}_{\Phi^{*}}^{*} H_{\Phi_{m}^{*}} \\
H_{\Phi_{m}^{*}}^{*} \mathbf{H}_{\Phi^{*}} & {\left[T_{\Phi_{m}}^{*}, T_{\Phi_{m}}\right]}
\end{array}\right] \geq 0
$$

or equivalently, for each $X \in \bigoplus_{j=1}^{m-1} H_{\mathbb{C}^{n}}^{2}$ and $Y \in H_{\mathbb{C}^{n}}^{2}$,

$$
\left|\left\langle\mathbf{H}_{\Phi^{*}} H_{\Phi_{m}^{*}}^{*} Y, X\right\rangle\right|^{2} \leq\left\langle\mathbf{H}_{\Phi^{*}}^{*} \mathbf{H}_{\Phi^{*}} X, X\right\rangle\left\langle\left[T_{\Phi_{m}}^{*}, T_{\Phi_{m}}\right] Y, Y\right\rangle .
$$


Since cl $\operatorname{ran} H_{\Phi_{i}^{*}}=\mathcal{H}\left(\widetilde{\Theta}_{i}\right)(i=1,2, \ldots, n-1)$, it follows that

$$
\begin{aligned}
\operatorname{cl} \operatorname{ran} \mathbf{H}_{\Phi^{*}} & =\bigvee_{i=1}^{m-1} \operatorname{cl} \operatorname{ran} H_{\Phi_{i}^{*}}=\bigvee_{i=1}^{m-1} \mathcal{H}\left(\widetilde{\Theta}_{i}\right)=\left(\bigcap_{i=1}^{m-1} \widetilde{\Theta}_{i} H_{\mathbb{C}^{n}}^{2}\right)^{\perp} \\
& =\left(\widetilde{\Theta} H_{\mathbb{C}^{n}}^{2}\right)^{\perp}=\mathcal{H}(\widetilde{\Theta})=\operatorname{cl} \operatorname{ran} H_{\Theta^{*}},
\end{aligned}
$$

where $\mathcal{H}(\Delta):=H_{\mathbb{C}^{n}}^{2} \ominus \Delta H_{\mathbb{C}^{n}}^{2}$. If the $\Phi_{i}$ are rational functions then, by (1.3) and (1.4), we can write

$$
\Phi_{i}=\theta_{i} A_{i}^{*} \quad\left(\theta_{i}, \text { finite Blaschke product }\right)
$$

Since $\Theta_{i}$ is a right inner divisor of $I_{\theta_{i}}$, we have $\operatorname{deg}\left(\Theta_{i}\right) \leq \operatorname{deg}\left(I_{\theta_{i}}\right)=n \operatorname{deg}\left(\theta_{i}\right)<\infty$. Thus since by (2.14), cl ran $\mathbf{H}_{\Phi^{*}}=\mathcal{H}(\widetilde{\Theta})$ and

$$
\operatorname{deg}(\Theta)=\operatorname{rank} H_{\Theta^{*}}^{*}=\operatorname{rank} H_{\Theta^{*}}=\operatorname{deg}(\widetilde{\Theta})<\infty
$$

Therefore $\mathbf{H}_{\Phi^{*}}$ is of finite rank and hence, so is $\mathbf{H}_{\Phi^{*}}^{*} \mathbf{H}_{\Phi^{*}}$ and, moreover,

$$
\operatorname{rank}\left(\mathbf{H}_{\Phi^{*}}^{*} \mathbf{H}_{\Phi^{*}}\right)=\operatorname{rank}\left(\mathbf{H}_{\Phi^{*}}^{*}\right)=\operatorname{rank}\left(\mathbf{H}_{\Phi^{*}}\right)=\operatorname{deg}(\Theta)
$$

Thus by (2.7), we have

$$
\begin{aligned}
\operatorname{rank}\left[\mathbf{T}^{*}, \mathbf{T}\right] & =\operatorname{rank}\left[\begin{array}{cc}
\mathbf{H}_{\Phi^{*}}^{*} \mathbf{H}_{\Phi^{*}} & \mathbf{H}_{\Phi^{*}}^{*} H_{\Phi_{m}^{*}} \\
H_{\Phi_{m}^{*}}^{*} \mathbf{H}_{\Phi^{*}} & {\left[T_{\Phi_{m}}^{*}, T_{\Phi_{m}}\right]}
\end{array}\right] \\
& =\operatorname{rank}\left(\mathbf{H}_{\Phi^{*}}^{*} \mathbf{H}_{\Phi^{*}}\right)+\operatorname{rank}\left(\left[T_{\Phi_{m}}^{*}, T_{\Phi_{m}}\right]-H_{\Phi_{m}^{*}}^{*} \mathbf{H}_{\Phi^{*}}\left(\mathbf{H}_{\Phi^{*}}^{*} \mathbf{H}_{\Phi^{*}}\right)^{\sharp} \mathbf{H}_{\Phi^{*}}^{*} H_{\Phi_{m}^{*}}\right) \\
& =\operatorname{deg}(\Theta)+\operatorname{rank}\left(\left[T_{\Phi_{m}}^{*}, T_{\Phi_{m}}\right]-H_{\Phi_{m}^{*}}^{*} \mathbf{H}_{\Phi^{*}}\left(\mathbf{H}_{\Phi^{*}}^{*} \mathbf{H}_{\Phi^{*}}\right)^{\sharp} \mathbf{H}_{\Phi^{*}}^{*} H_{\Phi_{m}^{*}}\right) .
\end{aligned}
$$

On the other hand, by Proposition 2.1, $\mathbf{H}_{\Phi^{*}}\left(\mathbf{H}_{\Phi^{*}}^{*} \mathbf{H}_{\Phi^{*}}\right)^{\sharp} \mathbf{H}_{\Phi^{*}}^{*}$ is the projection $P_{\mathcal{H}(\widetilde{\Theta})}$. Therefore it follows from (1.7) and (1.8) that

$$
\begin{aligned}
& {\left[T_{\Phi_{m}}^{*}, T_{\Phi_{m}}\right]-H_{\Phi_{m}^{*}}^{*} \mathbf{H}_{\Phi^{*}}\left(\mathbf{H}_{\Phi^{*}}^{*} \mathbf{H}_{\Phi^{*}}\right)^{\sharp} \mathbf{H}_{\Phi^{*}}^{*} H_{\Phi_{m}^{*}}} \\
& \quad=\left[T_{\Phi_{m}}^{*}, T_{\Phi_{m}}\right]-H_{\Phi_{m}^{*}}^{*} H_{\Theta^{*}} H_{\Theta^{*}}^{*} H_{\Phi_{m}^{*}} \\
& \quad=H_{\Phi_{m+}^{*}}^{*}\left(I-H_{\Theta^{*}} H_{\Theta^{*}}^{*}\right) H_{\Phi_{m+}^{*}}-H_{\Phi_{m-}^{*}}^{*} H_{\Phi_{m-}^{*}} \\
& \quad=\left(H_{\Phi_{m+}^{*}}^{*} T_{\widetilde{\Theta}}\right)\left(T_{\widetilde{\Theta}^{*}} H_{\Phi_{m+}^{*}}\right)-H_{\Phi_{m-}^{*}}^{*} H_{\Phi_{m-}^{*}} \\
& \quad=H_{\Theta \Phi_{m+}^{*}}^{*} H_{\Theta \Phi_{m+}^{*}}-H_{\Phi_{m-}^{*}}^{*} H_{\Phi_{m-}^{*}} \\
& \quad=\left[T_{\Phi_{m}^{*}}^{*, \Theta}, T_{\Phi_{m}^{1, \Theta}}\right]_{p},
\end{aligned}
$$

which gives the result.

Very recently, the hyponormality of rational Toeplitz pairs was characterized in [16]. 
Lemma 2.7 (Hyponormality of rational Toeplitz pairs) [16] Let $\mathbf{T} \equiv\left(T_{\Phi}, T_{\Psi}\right)$ be a Toeplitz pair with rational symbols $\Phi, \Psi \in L_{M_{n}}^{\infty}$ of the form

$$
\Phi_{+}=\theta_{0} \theta_{1} A^{*}, \quad \Phi_{-}=\theta_{0} B^{*}, \quad \Psi_{+}=\theta_{2} \theta_{3} C^{*}, \quad \Psi_{-}=\theta_{2} D^{*} \quad \text { (coprime). }
$$

Assume that $\theta_{0}$ and $\theta_{2}$ are not coprime. Assume also that $B\left(\gamma_{0}\right)$ and $D\left(\gamma_{0}\right)$ are diagonalconstant for some $\gamma_{0} \in \mathcal{Z}\left(\theta_{0}\right)$. Then the pair $\mathbf{T}$ is hyponormal if and only if

(i) $\Phi$ and $\Psi$ are normal and $\Phi \Psi=\Psi \Phi$;

(ii) $\Phi_{-}=\Lambda^{*} \Psi_{-}\left(\right.$with $\left.\Lambda:=B\left(\gamma_{0}\right) D\left(\gamma_{0}\right)^{-1}\right)$;

(iii) $T_{\Psi^{1, \Omega}}$ is pseudo-hyponormal with $\Omega:=\theta_{0} \theta_{1} \theta_{3} \bar{\theta} \Delta^{*}$,

where $\theta:=$ g.c.d. $\left(\theta_{1}, \theta_{3}\right)$ and $\Delta:=$ left-g.c.d. $\left(I_{\theta_{0} \theta}, \bar{\theta}\left(\theta_{3} A-\theta_{1} C \Lambda^{*}\right)\right)$.

We now get a rank formula for the self-commutators of Toeplitz $m$-tuples.

Corollary 2.8 For each $i=1,2, \ldots, m$, suppose that $\Phi_{i}=\left(\Phi_{i}\right)_{-}^{*}+\left(\Phi_{i}\right)_{+} \in L_{M_{n}}^{\infty}$ is a matrixvalued normal rational function of the form

$$
\left(\Phi_{i}\right)_{+}=\theta_{i} \delta_{i} A_{i}^{*} \quad \text { and } \quad\left(\Phi_{i}\right)_{-}=\theta_{i} B_{i}^{*} \quad(\text { coprime })
$$

where the $\theta_{i}$ and the $\delta_{i}$ are finite Blaschke products and there exists $j_{0}\left(1 \leq j_{0} \leq m\right)$ such that $\theta_{j_{0}}$ and $\theta_{i}$ are not coprime for each $i=1,2, \ldots, m$. Suppose $\Phi_{i} \Phi_{j}=\Phi_{j} \Phi_{i}$ for all $i, j=1, \ldots, m$. Assume that each $B_{i}\left(\gamma_{0}\right)$ is diagonal-constant for some $\gamma_{0} \in \mathcal{Z}\left(\theta_{i}\right)$. If $\mathbf{T} \equiv\left(T_{\Phi_{1}}, T_{\Phi_{2}}, \ldots, T_{\Phi_{m}}\right)$ is hyponormal then

$$
\operatorname{rank}\left[\mathbf{T}^{*}, \mathbf{T}\right]=\operatorname{deg}(\Omega)+\operatorname{rank}\left[T_{\Phi_{j_{0}}^{1, \Omega}}^{*}, T_{\Phi_{j_{0}}^{1, \Omega}}\right]_{p},
$$

where $\Omega:=$ right-l.c.m. $\left\{\theta_{i} \delta_{i} \delta_{j_{0}} \overline{\delta(i)} \Theta(i)^{*}: i=1,2, \ldots, m\right\}$. Here $\delta(i):=$ g.c.d. $\left\{\delta_{i}, \delta_{j_{0}}\right\}$ and $\Theta(i):=$ left-g.c.d. $\left\{\theta_{i} \delta(i), \overline{\delta(i)}\left(\delta_{j_{0}} A_{i}-\delta_{i} A_{j_{0}} \Lambda(i)^{*}\right)\right\}$ with $\Lambda(i):=B_{i}\left(\gamma_{0}\right) B_{j_{0}}\left(\gamma_{0}\right)^{-1}$.

Proof Suppose $\mathbf{T}$ is hyponormal. Since every sub-tuple of $\mathbf{T}$ is hyponormal, we can see that $\left(T_{\Phi_{i}}, T_{\Phi_{j}}\right)$ is hyponormal for all $i, j=1,2, \ldots, m$. In view of $(2.10)$, we may assume that $j_{0}=m$. Put

$$
\mathbf{S}:=\left(T_{\Phi_{1}-\Lambda(1) \Phi_{m}}, T_{\Phi_{2}-\Lambda(2) \Phi_{m}}, \ldots, T_{\Phi_{m-1}-\Lambda(m-1) \Phi_{m}}, T_{\Phi_{m}}\right) .
$$

It follows from Corollary 2.5 that

$\mathbf{T}$ is hyponormal $\Longleftrightarrow \mathbf{S}$ is hyponormal.

Since $\delta(i)=$ g.c.d. $\left\{\delta_{i}, \delta_{m}\right\}$, we can write

$$
\delta_{i}=\delta(i) \omega_{i} \quad \text { and } \quad \delta_{m}=\delta(i) \omega_{m}
$$

where $\omega_{i}$ is a finite Blaschke product for $i=1,2, \ldots, m$. Since $\Theta(i)=$ left-g.c.d. $\left\{\theta_{i} \delta(i)\right.$, $\left.\overline{\delta(i)}\left(\delta_{m} A_{i}-\delta_{1} A_{m} \Lambda(i)^{*}\right)\right\}$, we get the following left coprime factorization:

$$
\Phi_{i}-\Lambda(i) \Phi_{m}=\left[\left(\overline{\omega_{m}} A_{i}^{*}-\overline{\omega_{i}} \Lambda(i) A_{m}^{*}\right) \Theta(i)\right] \theta_{i} \delta_{i} \delta_{m} \overline{\delta(i)} \Theta(i)^{*}
$$

Thus the result follows at once from Theorem 2.6. 
We conclude with the following.

Corollary 2.9 For each $i=1,2, \ldots, m$, suppose that $\phi_{i}=\overline{\left(\phi_{i}\right)_{-}}+\left(\phi_{i}\right)_{+} \in L^{\infty}$ is a rational function of the form

$$
\left(\phi_{i}\right)_{+}=\theta_{i} \overline{a_{i}} \text { and }\left(\phi_{i}\right)_{-}=\theta_{i} \overline{b_{i}} \quad \text { (coprime). }
$$

If there exists $j_{0}\left(1 \leq j_{0} \leq m\right)$ such that $\theta_{j_{0}}$ and $\theta_{i}$ are not coprime for each $i=1,2, \ldots, m$ and $\mathbf{T} \equiv\left(T_{\phi_{1}}, T_{\phi_{2}}, \ldots, T_{\phi_{m}}\right)$ is hyponormal then

$$
\operatorname{rank}\left[\mathbf{T}^{*}, \mathbf{T}\right]=\operatorname{rank}\left[T_{\Phi_{j_{0}}}^{*}, T_{\Phi_{j_{0}}}\right]
$$

Proof For each $i=1,2, \ldots, m$, let $\lambda(i):=b_{i}\left(\gamma_{0}\right) b_{j_{0}}\left(\gamma_{0}\right)^{-1}$ for some $\gamma_{0} \in \mathcal{Z}\left(\theta_{i}\right)$. Write $\theta(i) \equiv$ g.c.d. $\left\{\theta_{i},\left(a_{i}-a_{j_{0}} \overline{\lambda(i)}\right)\right\}$. Since $\mathbf{T} \equiv\left(T_{\phi_{1}}, T_{\phi_{2}}, \ldots, T_{\phi_{n}}\right)$ is hyponormal, $\left(T_{\phi_{i}}, T_{\phi_{j_{0}}}\right)$ is hyponormal for all $i=1,2, \ldots, n$. Thus it follows from Lemma 2.7 that $T_{\phi_{j}^{1, \omega(i)}}$ is hyponormal with $\omega(i):=\theta_{i} \overline{\theta(i)}$. Observe that

$$
\left(\phi_{j_{0}}^{1, \omega(i)}\right)_{+}=\theta(i) \overline{c_{i}} \quad \text { and } \quad\left(\phi_{j_{0}}^{1, \omega(i)}\right)_{-}=\theta_{i} \overline{b_{i}} \quad \text { (coprime) }
$$

where $c_{i}:=P_{\mathcal{H}(\theta(i))}\left(a_{i}\right)$. Since $T_{\phi_{j}^{1, \omega(i)}}$ is hyponormal, it follows from Proposition 2.2 that $\theta_{i}$ is an inner divisor of $\theta(i)$ and hence $\theta(i)=\theta_{i}$. Thus the result follows from Corollary 2.8.

\title{
3 Conclusions
}

The self-commutators of bounded linear operators play an important role in the study of hyponormal and subnormal operators. The main result of this paper is to derive a rank formula for the self-commutators of tuples of Toeplitz operators with matrix-valued rational symbols. This result will contribute to the study of Toeplitz operators and the bridge theory of operators.

\section{Competing interests}

The authors declare that they have no competing interests.

\section{Authors' contributions}

The authors contributed equally and significantly in writing this paper.

\author{
Author details \\ 'Department of Mathematics, Sungkyunkwan University, Suwon, 440-746, Korea. ${ }^{2}$ Department of Mathematics, \\ Changwon National University, Changwon, 641-773, Korea.
}

\section{Acknowledgements}

The work of the first author was supported by National Research Foundation of Korea (NRF) grant funded by the Ministry of Education, Science and Technology (No. 2011-0022577). The work of the second author was supported by Basic Science Research Program through the National Research Foundation of Korea (NRF) funded by the Ministry of Education (No. 2015R1D1A3A01016258)

Received: 14 April 2016 Accepted: 2 July 2016 Published online: 22 July 2016

\section{References}

1. Böttcher, A, Silbermann, B: Analysis of Toeplitz Operators. Springer, Berlin (2006)

2. Douglas, RG: Banach Algebra Techniques in Operator Theory. Academic Press, New York (1972)

3. Nikolskii, NK: Treatise on the Shift Operator. Springer, New York (1986)

4. Peller, W: Hankel Operators and Their Applications. Springer, New York (2003)

5. Abrahamse, MB: Subnormal Toeplitz operators and functions of bounded type. Duke Math. J. 43, 597-604 (1976) 
6. Gu, C, Hendricks, J, Rutherford, D: Hyponormality of block Toeplitz operators. Pac. J. Math. 223, 95-111 (2006)

7. Curto, RE, Hwang, IS, Lee, WY: Hyponormality and subnormality of block Toeplitz operators. Adv. Math. 230, 2094-2151 (2012)

8. Curto, RE, Hwang, IS, Lee, WY: Which subnormal Toeplitz operators are either normal or analytic? J. Funct. Anal. 263(8), 2333-2354 (2012)

9. Bram, J: Subnormal operators. Duke Math. J. 22, 75-94 (1955)

10. Conway, JB: The Theory of Subnormal Operators. Math Surveys and Monographs, vol. 36. Am. Math. Soc., Providence (1991)

11. Athavale, A: On joint hyponormality of operators. Proc. Am. Math. Soc. 103, 417-423 (1988)

12. Curto, RE, Muhly, PS, Xia, J: Hyponormal pairs of commuting operators. In: Gohberg, I, Helton, JW, Rodman, L (eds.): Contributions to Operator Theory and Its Applications, Mesa, AZ, 1987. Operator Theory: Advances and Applications, vol. 35, pp. 1-22. Birkhäuser, Basel (1988)

13. Harte, RE: Invertibility and Singularity for Bounded Linear Operators. Monographs and Textbooks in Pure and Applied Mathematics, vol. 109. Dekker, New York (1988)

14. Curto, RE, Lee, WY: Joint Hyponormality of Toeplitz Pairs. Memoirs Amer. Math. Soc., vol. 712. Am. Math. Soc., Providence (2001)

15. Gu, C: On a class of jointly hyponormal Toeplitz operators. Trans. Am. Math. Soc. 354, 3275-3298 (2002)

16. Curto, RE, Hwang, IS, Lee, WY: Matrix functions of bounded type: An interplay between function theory and operator theory. Preprint (2016)

\section{Submit your manuscript to a SpringerOpen ${ }^{\circ}$ journal and benefit from:}

- Convenient online submission

- Rigorous peer review

- Immediate publication on acceptance

- Open access: articles freely available online

- High visibility within the field

- Retaining the copyright to your article 